\title{
Early Prenatal Attainment of Adult Metacarpal-Phalangeal Rankings and Proportions
}

\author{
STANLEY M. GARN, ALPHONSE R. BURDI, WILLIAM J. BABLER \\ AND SARA STINSON \\ The Center for Human Growth and Development, and Department of \\ Anatomy, The University of Michigan, Ann Arbor, Michigan 48104
}

KEY WORDS Metacarpals - Phalanges · Proportions - Bone-tobone ratios $\cdot$ Embryology $\cdot$ Fetal development $\cdot$ Histology.

\begin{abstract}
As shown in 56 human embryos and fetuses between 15 and $104 \mathrm{~mm}$ in crown-rump length, "adult" metacarpal-phalangeal length rankings are attained by the seventh intrauterine week and near-adult bone-to-bone ratios or proportions by the thirteenth week. Micrometric measurements of optically-projected histological hand sections show relative elongation of the distals between the $15-29 \mathrm{~mm}$ and $30-44 \mathrm{~mm}$ crown-rump range, and relative reduction to radiogrammetrically-determined adult proportions by the 90 $104 \mathrm{~mm}$ CRL.
\end{abstract}

Although numerous qualitative parameters of prenatal hand development have been explored by human embryologists and developmental anatomists, little attention has been devoted to the origins of hand shape and to the relative lengths of the 19 metacarpals and phalanges during embryonic and fetal development. The older literature contains many studies of carpal differentiation, with special reference to the developmental origins of carpal and metacarpal "fusions," while the more recent literature relates to the onset of calcification of the tubular bones of the hand (cf. Jit, '70) and to ossification of the tubular bones as seen in radiographs (cf. Kjaer, '74). Yet photomicrographs of embryonic and fetal hands show a surprising resemblance to the adult hand in relative metacarpal and phalangeal lengths and proportions (cf. Garn et al., '74; fig. 1).

We have therefore assembled complete histological hand sections of 56 normal human embryos and fetuses of known sex and crown-rump length, and we have measured lengths of individual metacarpals, proximals, middles and distals in embryos and fetuses between 15 and $104 \mathrm{~mm}$ in crown-rump length. These measurements, made in optical projections of the mounted histological sections, show early attainment of relative lengths closely resembling adult standards we have previously pub- lished (cf. Garn et al., '72). Bone-to-bone ratios or proportions derived from these measurements evidence a very early disproportionate elongation of distals, followed by a gradual approximation of adult proportions by the 90-104 $\mathrm{mm}$ crown-rump length.

\section{METHODS AND MATERIALS}

The present study is based upon verniercaliper measurements of optically-projected images of 5-10 $\mu$ sections of the hands of 56 embryos and fetuses between 15 and $104 \mathrm{~mm}$ crown-rump length (CRL). All human specimens used in this investigation were sex-identified either by direct inspection of external genitalia or (in the younger specimens) by microscopic examination of gonadal development. Specimens with gross or histological defects, or with hand malformations were excluded from this developmental study.

For each embryo or fetus, serial microscopic slides were placed directly on the stage of a Leitz vertical projection microscope ("Projektiskop") and the opticallyprojected images were measured with a Helios INOXYO vernier micrometer with $0.1 \mathrm{~mm}$ readout. Actual enlargement ratios, using parfocalized objectives selected according to individual bone lengths, were ascertained with an American Optical stage micrometer (Cat. No. 1400). Maxi- 


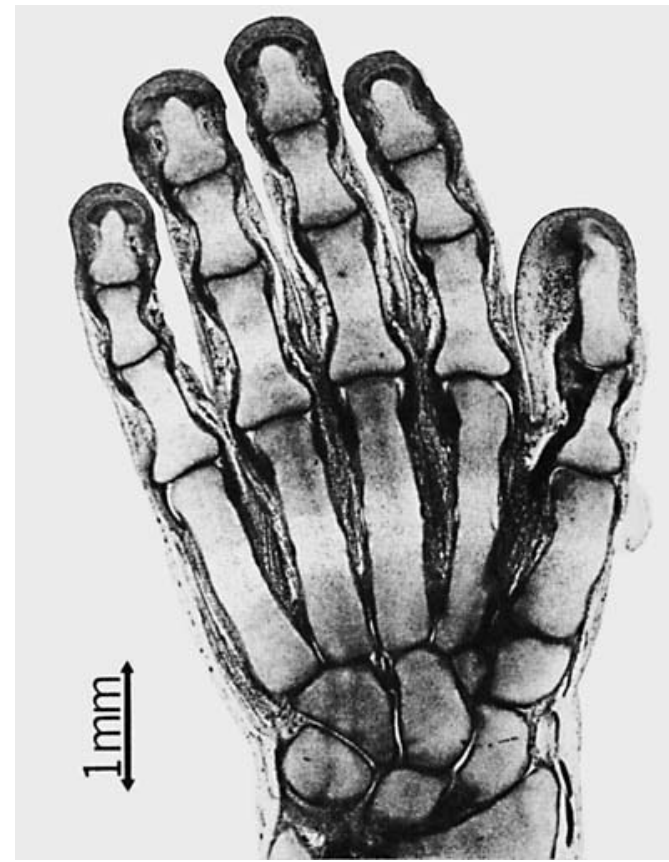

Fig. 1 Photomicrograph of the hand of a male fetus of $47 \mathrm{~mm}$ crown-rump length (CRL) showing in a single histological section the lengths of nearly all metacarpals, proximals, middles and distals, along with a $1 \mathrm{~mm}$ reference scale. Individual bone length of this typical male fetus evidence a 0.94 rank-order correlation (rho) with the adult bone length norms employed, and a 0.98 rankorder correlation with median bone lengths for the $45-59 \mathrm{~mm}$ crown-rump length grouping. For information on dimensional stability of specimens prepared with $10 \%$ neutral buffered formalin see Patten and Philpott ('21).

mum lengths of individual bones were measured, in the method described by Garn et al. (72) for radiographic images. In many cases, multiple histological sections were employed to ascertain maximum individual bone lengths, as determined by a single operator (WJB) throughout. Separate analyses of inter-observer reliability showed such reliability coefficients in excess of 0.98 , RMS measuring errors of the order of 0.05 for larger specimens, and maximum measuring errors under $2 \%$, even when different sections were involved.

In the first part of the data analysis, metacarpal and phalangeal lengths were calculated as median values, for each of six crown-rump lengths, grouped into 15 $\mathrm{mm}$ CRL intervals, from 15-29 $\mathrm{mm}$ through 90-104 mm CRL. These median values, for each metacarpal, proximal, middle and distal were compared with adult lengths as given by Garn et al. ('72) using rankorder correlations (rho), Fifty-six individual values of rho were similarly calculated.

In the second part of the data analysis, bone-to-bone ratios or proportions were also calculated, both for each specimen and for the six CRL groupings as described above. These bone-to-bone ratios or proportions were then compared with adult bone-tobone ratios similarly calculated by us and recently reprinted in Poznanski ('74).

These two procedures allowed the comparison of metacarpal and phalangeal lengths and length rankings, from the 15 through $104 \mathrm{~mm}$ crown-rump lengths, with adult lengths and length rankings. They also allowed comparison of metacarpalphalangeal bone-to-bone ratios or proportions, over the range of crown-rump lengths considered and in comparison to adult bone-to-bone ratios similarly calculated.

\section{FINDINGS}

In the first step of the data analysis, median values for each of 19 metacarpals and phalanges were separately calculated for each of six equal-interval crown-rump length groupings, and for males and females respectively. Since no systematic sex difference was found, in comparing median metacarpal and phalangeal lengths, male and female data were recalculated as combined-sex median values. These are shown for the 15-29 mm, 30-44 mm, 45$59 \mathrm{~mm}, 60-74 \mathrm{~mm}, 75-89 \mathrm{~mm}$ and $90-$ $104 \mathrm{~mm}$ crown-rump groupings in table 1.

In the second step of analysis, fetal metacarpal and phalangeal lengths were compared with radiogrammetrically-determined adult length values for the same bones, as given in Garn et al. (72) and reprinted in Poznanski (74). Comparisons were then made, for each of the six crown-rump length groupings, by ranking median measurements from highest (19) to lowest (1) and calculating the rank-order correlation (rho). Bone-to-bone rankings both for the fetal bone lengths and the adult bone lengths are shown in table 1 , as are the rank order correlations (rho). In a general way, fetal bone rankings corresponded to the adult length rankings in the same bones. For particular crown-rump length 


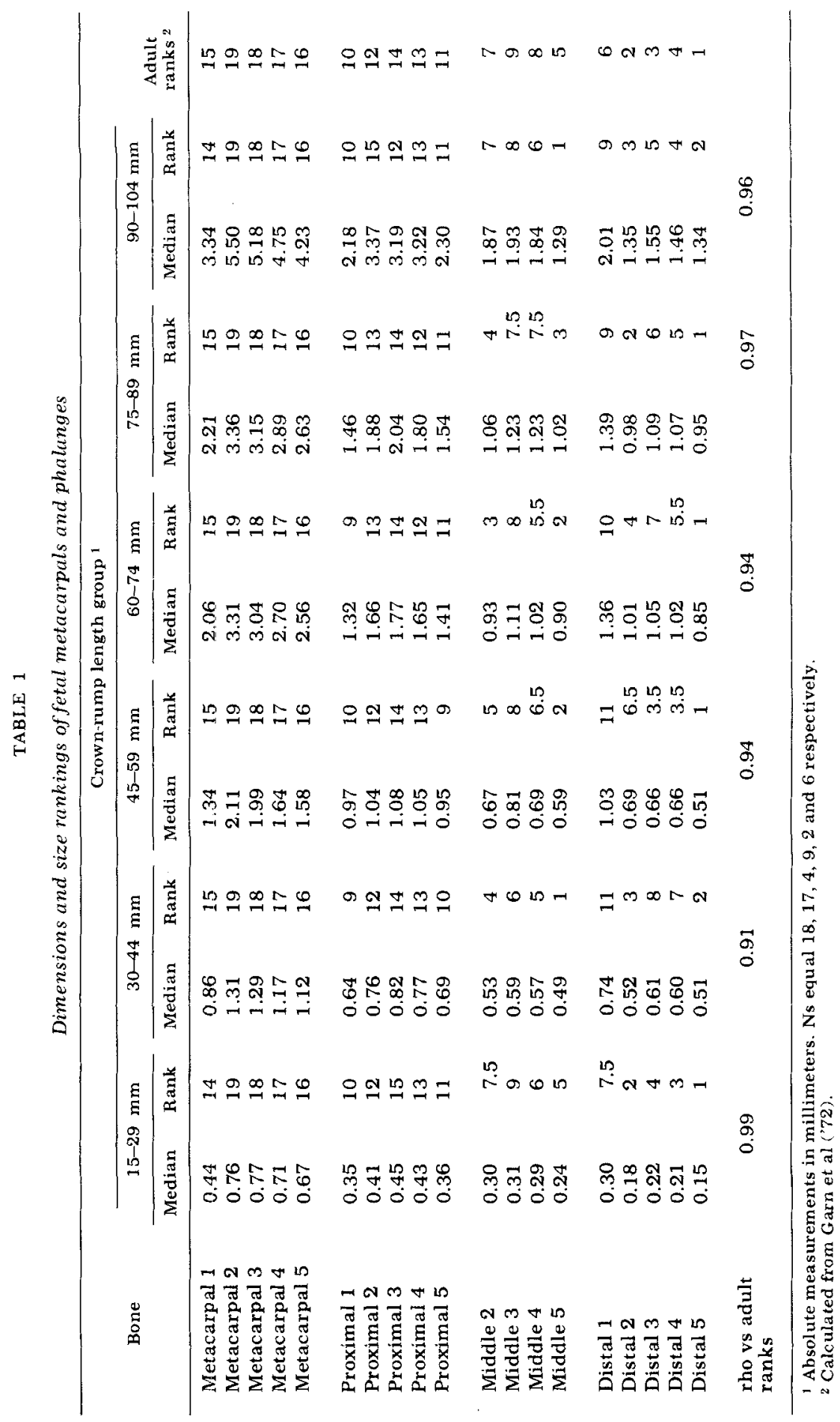


groupings, values of rho varied from 0.91 to 0.99 , with an overall value of rho of 0.95 . In general, metacarpals agreed closely with their adult rankings $(15,19,18$, 17 and 16) from the first through the fifth metacarpal, whereas somewhat greater deviations characterized the distals and the middle segment of the fifth digit. Overall, then, metacarpal and phalangeal length rankings in the 56 fetuses showed good approximation to the adult length rankings, somewhat less so for the five distals and mid $\mathrm{V}$.

In the third step of the data analysis, attention was given to bone-to-bone ratios, or proportions, uniformly calculated as described in Poznanski (74). Since the complete matrix of bone-to-bone ratios includes some 171 different ratios or proportions, the data presentation was simplified by expressing the proportions of each bone relative to the second metacarpal, invariably the longest bone of all. This approach was used both for the fetuses (excluding 3 specimens where histologic sections of the second metacarpal were technically unsatisfactory) and for the adult hand values, computer recalculated and checked for the present investigation.
As shown in the second table, fetal boneto-bone ratios also show reasonable correspondence to adult bone-to-bone ratios, similarly calculated. In the largest (90$104 \mathrm{~mm}$ ) crown-rump grouping, 12 of the 18 ratios were within \pm 1 SD of the adult (female) bone-to-bone ratios, using largesample adult values of $\sigma$. Even in the smallest (15-29 mm) crown-rump length grouping, half of the 18 bone-to-bone ratios were within \pm 1 SD of the adult (female) boneto-bone ratios. Given the fact that the individual bones involved were all $1.0 \mathrm{~mm}$ or less in length, and 11 were below 0.5 $\mathrm{mm}$ in length at the 15-29 $\mathrm{mm}$ crownrump grouping, these comparabilities in fetal vs. adult metacarpal-phalangeal proportions are remarkable.

However, careful attention to table 2, and the bone-to-bone ratios given therein, evidences a novel and major discovery about early fetal metacarpal and phalangeal growth. Though at 15-29 $\mathrm{mm}$, and again at $90-104 \mathrm{~mm}$, relative size of the distals reasonably approximates that in the adult hand, there is a rapid and disproportionate growth of distals between the 15-29 and the $30-44 \mathrm{~mm}$ crown-rump range, diminishing thereafter.

TABLE 2

Bone-to-bone ratios in adults and in the fetal period

\begin{tabular}{|c|c|c|c|c|c|c|c|c|}
\hline \multirow{2}{*}{ Bone } & \multicolumn{6}{|c|}{$\begin{array}{l}\text { Ratios relative to metacarpal } 2 \\
\text { Fetal ratios by crown-rump length } 1\end{array}$} & \multicolumn{2}{|c|}{$\begin{array}{l}\text { Adult } \\
\text { ratios } 2\end{array}$} \\
\hline & $15-29 \mathrm{~mm}$ & $30-44 \mathrm{~mm}$ & $45-59 \mathrm{~mm}$ & $60-74 \mathrm{~mm}$ & $75-89 \mathrm{~mm}$ & $90-104 \mathrm{~mm}$ & Mean & S.D. \\
\hline Metacarpal 1 & 0.60 & 0.66 & 0.68 & 0.61 & 0.66 & 0.67 & 0.66 & 0.03 \\
\hline Metacarpal 2 & $\longrightarrow$ & - & $\longrightarrow$ & - & - & - & - & $\underline{-}$ \\
\hline Metacarpal 3 & 1.00 & 0.98 & 0.96 & 0.94 & 0.94 & 0.94 & 0.94 & 0.02 \\
\hline Metacarpal 4 & 0.93 & 0.91 & 0.87 & 0.84 & 0.86 & 0.86 & 0.84 & 0.03 \\
\hline Metacarpal 5 & 0.90 & 0.82 & 0.82 & 0.80 & 0.78 & 0.79 & 0.78 & 0.05 \\
\hline Proximal 1 & 0.46 & 0.51 & 0.49 & 0.44 & 0.44 & 0.43 & 0.47 & 0.03 \\
\hline Proximal 2 & 0.55 & 0.61 & 0.53 & 0.52 & 0.59 & 0.61 & 0.60 & 0.03 \\
\hline Proximal 3 & 0.59 & 0.64 & 0.57 & 0.60 & 0.61 & 0.63 & 0.66 & 0.04 \\
\hline Proximal 4 & 0.60 & 0.60 & 0.55 & 0.55 & 0.53 & 0.59 & 0.61 & 0.03 \\
\hline Proximal 5 & 0.46 & 0.52 & 0.48 & 0.45 & 0.46 & 0.46 & 0.49 & 0.03 \\
\hline Middle 2 & 0.37 & 0.39 & 0.34 & 0.31 & 0.31 & 0.33 & 0.35 & 0.02 \\
\hline Middle 3 & 0.38 & 0.46 & 0.38 & 0.33 & 0.37 & 0.38 & 0.42 & 0.03 \\
\hline Middle 4 & 0.38 & 0.40 & 0.35 & 0.31 & 0.37 & 0.36 & 0.40 & 0.02 \\
\hline Middle 5 & 0.31 & 0.33 & 0.30 & 0.27 & 0.30 & 0.25 & 0.28 & 0.02 \\
\hline Distal 1 & 0.35 & 0.55 & 0.50 & 0.44 & 0.41 & 0.36 & 0.33 & 0.02 \\
\hline Distal 2 & 0.22 & 0.40 & 0.34 & 0.31 & 0.29 & 0.28 & 0.25 & 0.02 \\
\hline Distal 3 & 0.27 & 0.42 & 0.30 & 0.30 & 0.32 & 0,28 & 0.26 & 0.02 \\
\hline Distal 4 & 0.26 & 0.40 & 0.33 & 0.29 & 0.32 & 0.28 & 0.27 & 0.02 \\
\hline Distal 5 & 0.20 & 0.35 & 0.28 & 0.25 & 0.28 & 0.24 & 0.24 & 0.02 \\
\hline
\end{tabular}

1 Ns equal 17, 16, 4, 9, 2 and 5 respectively. Ratios expressed as a decimal fraction of metacarpal 2.

2 Female ratios as given in Poznanski ('74), since female variances are slightly greater. 
Such disproportionate early growth of the five distals between the first two crownrump length groupings is of such magnitude as to make them relatively far longer in the $30-44 \mathrm{~mm}$ crown-rump range than at any other developmental horizon. Though still "short" in absolute millimeter measurements, compared with metacarpals or most proximals, the relative lengths of distals 1-5 are larger at 30-44, 45-59, 60-74, and 75-89 $\mathrm{mm}$, compared with adult bone-to-bone ratios or proportions. However, as shown in figure 2 , the relative lengths of distals 1-5 diminish progressively from the second through the sixth crown-rump grouping.

Using size rankings, therefore, it is clear that the tubular bones of the hand that are longer in the adult are already the longer bones even in the 15-29 mm embryos, and through the $90-104 \mathrm{~mm}$ fetal crownrump range. Even in the 15-29 $\mathrm{mm}$ em-

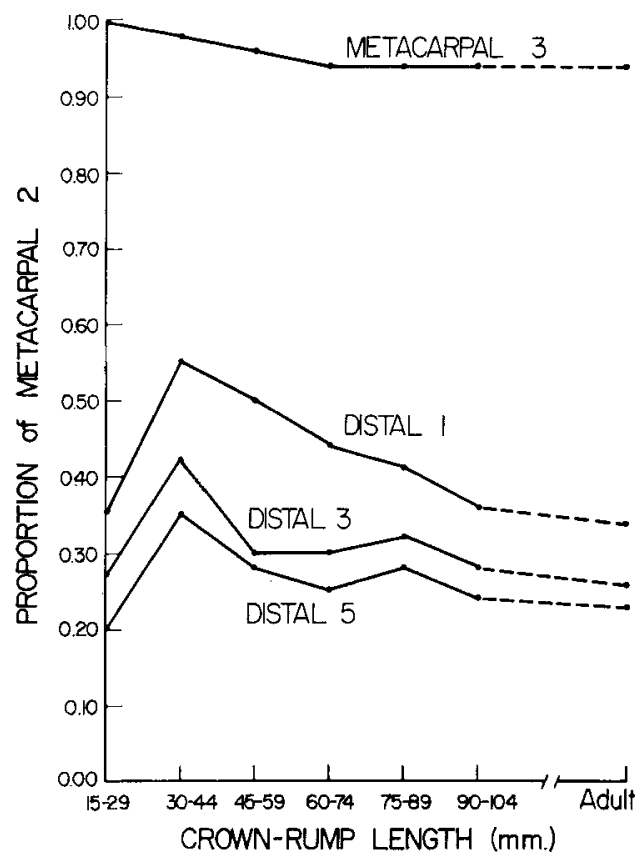

Fig. 2 Changing bone-to-bone ratios shown for metacarpal III and distals 1,3 and 5, in six crownrump length groupings and in the adult norms. As shown by reference to the second metacarpal (uniformly the longest of the 19 tubular bones of the hand), the distals undergo relative elongation between the $29 \mathrm{~mm}$ and $30-40 \mathrm{~mm}$ crown-rump lengths, primarily due to tuft development, and relative decrease thereafter, closely approximating adult relative lengths by the $90-104 \mathrm{~mm}$ crownrump range. bryo, the distals are the shortest five bones of the hand and the five metacarpals closely approximate their adult size rankings. Adult metacarpal and phalangeal rankings are demonstrably attained early, by the second month of gestation, at least.

At the same time, or during the same period, the distals undergo transient relative length hypertrophy. Though still short bones, compared with metacarpals or proximals, the distals become far longer (compared with the metacarpals) in the 30 $44 \mathrm{~mm}$ crown-rump range and diminish in relative size through to the $90-104 \mathrm{~mm}$ crown-rump range.

\section{DISCUSSION}

As shown in 56 human embryos and fetuses, all sex-identified and all histologically-normal, adult metacarpal and phalangeal length rankings are attained remarkably early. For all practical purposes, in fact, adult length rankings of the 19 metacarpals, proximals, middles and distals are attained by the seventh intrauterine week. Using both individual length rankings, for each specimen, and rankings based on median length values for each of the six crown-rump length groupings, correspondences with adult radiogrammetric rankings are considerable, approximating 0.95 overall. It would appear, therefore, that relative metacarpal and phalangeal lengths characteristic of the adult are established in the mesenchymal and cartilaginous human hand models well before initial bone-collar and distal-tuft calcification begins.

These studies, moreover, based on direct optical-projection measurements of 5-10 $\mu$ histological sections, evidence a remarkable relative elongation of the distal segments between the 15-29 and 30-44 mm crown-rump groupings. In the $15-29 \mathrm{~mm}$ crown-rump range relative lengths of the distals, as shown by bone-to-bone ratios, are near-adult proportions or ratios. In the $30-44 \mathrm{~mm}$ crown-rump length groupings, all five distals are far longer relative to metacarpal 2 or other metacarpals and proximals. Thereafter, relative distal lengths decrease, for all five distals, until by the 90-104 $\mathrm{mm}$ crown-rump length they again approximate the adult bone-tobone ratios individually calculated from radiogrammetric lengths (cf. Poznanski, '74). By the thirteenth intrauterine week, 
therefore, adult length rankings and adult bone-to-bone proportions are both attained.

It would appear, therefore, that the eighth-ninth intrauterine week is a critical period for distal elongation, and the tenth through thirteenth weeks are similarly critical to relative or proportional distal reduction. It might also be suggested that the adult hand model is established early, on an individual as well as a group basis, with the rankings and proportions at 13 weeks individually predictive of adult metacarpal-phalangeal proportions.

We can not, of course, confirm this latter suggestion by serial, longitudinal studies of individuals beginning with the 15$29 \mathrm{~mm}$ crown-rump range and continuing through metacarpal-phalangeal length completion. We can, however, demonstrate metacarpal-phalangeal length and proportional concordance in fetal twins, and to a lesser extent in fetal (singling) siblings. Moreover, adult-parent vs. fetal metacarpal-phalangeal length, proportion and pattern comparisons and older-sibling vs. fetal metacarpal-phalangeal comparisons are within the realm of practicability. Such designs, coupled with analysis of fetuses with malformation syndromes may establish the extent to which the fetal hand individually predicts adult metacarpal-phalangeal proportions.

\section{ACKNOWLEDGMENTS}

The work described in this paper, utilizing embryos and fetuses catalogued within the Patten Embryological Research Collection of The University of Michigan was supported, in part, by grant DE-03443 from the National Institute of Dental Research, and by a stipend from the Beatrice Stanton Medical Research Fund of The University of Michigan School of Medicine. We appreciate the assistance of Miss Dixie Farquharson in manuscript completion.

\section{LITERATURE CITED}

Garn, S. M., A. R. Burdi and W. J. Babler 1974 Male advancement in prenatal hand development. Am. J. Phys. Anthrop., 41: 353-360.

Garn, S. M., K. P. Hertzog, A. K. Poznanski and J. M. Nagy 1972 Metacarpophalangeal length in the evaluation of skeletal malformation. Radiology, 105: 375-381.

Gefferth, K. 1972 Metrische Auswertung der kurzen Röhrenknochen der Hand von der $\mathrm{Ge}$ burt bis zum Ende der Pubertät: Längenmasse. Acta Paediat. Acad. Sci. Hung., 13: 117-124.

Jit, I. 1970 Observations on prenatal ossifica tion with special reference to the bones of the hand and foot. J. Anat. Soc. India, $6: 12-23$.

Kjaer, I. 1974 Skeletal maturation of the human fetus assessed radiographically on the basis of ossification sequences in the hand and foot Am. J. Phys. Anthrop., 40: 257-275.

Patten, B. M., and R. Philpott 1921 The shrink age of embryos in the process preparatory to sectioning. Anat. Rec., 20: 393-413.

Poznanski, A. K. 1974 The Hand in Radiographic Diagnosis. Saunders and Co., New York. 